\title{
Letting off steam: the Stockholm Message from World Water Week to the COP-15
}

\author{
Karin Lexén · Alan Nicol · Henk van Schaik
}

Published online: 14 November 2009

(C) Springer Science+Business Media B.V. 2009

In the closing plenary session of the recent 2009 Stockholm World Water Week participants unanimously voted to support the Stockholm Message on Water and Climate to the COP-15 (the 15th Conference Of the Parties to the United Framework Convention on Climate Change). This became the Stockholm Statement which was subsequently forwarded to many Stockholm International Water Institute partners and governmental delegations to the climate negotiations.

\section{The climate kettle}

With each year, we witness new evidence that the planet is warming up. A body of established scientific evidence-all but irrefutable except to hardened sceptics-points to a global increase of at least $2^{\circ} \mathrm{C}$, by the end of this century. Some evidence leads to

K. Lexén $(\bowtie)$

Stockholm International Water Institute,

Stockholm, Sweden

e-mail: karin.lexen@siwi.org

\author{
A. Nicol \\ World Water Council, Marseilles, France \\ e-mail: a.nicol@wwc.org \\ H. van Schaik \\ Co-operative Programme on Water and Climate, \\ The Hague, The Netherlands \\ e-mail: h.vanschaik@cpwc.nl
}

even more dire predictions of a $4^{\circ}$ rise, and perhaps even more by mid to late century.

Any high school science student knows that heating water increases the volatility of the liquid, turning it into vapour, or steam. As the planet warms up, this will be one of the certain impacts-the speeding up of the hydrological cycle. Even so, we don't know much about how that vapour will behave, where it will condense and form rain, and how much will evaporate back again into the atmosphere. This confronts humanity with a simple but critical question: What will happen to the water cycle, the planetary ‘bloodsystem'? Fig. 1.

The positive and negative feedback loops that comprise our global climate system are so complex that we are only in the infancy of effective climate prediction and modeling. We know that something will happen, but predicting the what, where and when at a scale that helps countries and regions to prepare responses is still some way off.

Acquiring knowledge about impacts is also the beginning of the story, not the end. What to do with that knowledge is a major challenge and one that confronts water managers and political leaders tasked with decision-making on future investments and policy. Increasingly these decisions will need to be based on higher levels of confidence. As the science-and knowledge of that science-improves this will help the development and effective implementation of well-designed policies and strategies on water management, and wider issues including 


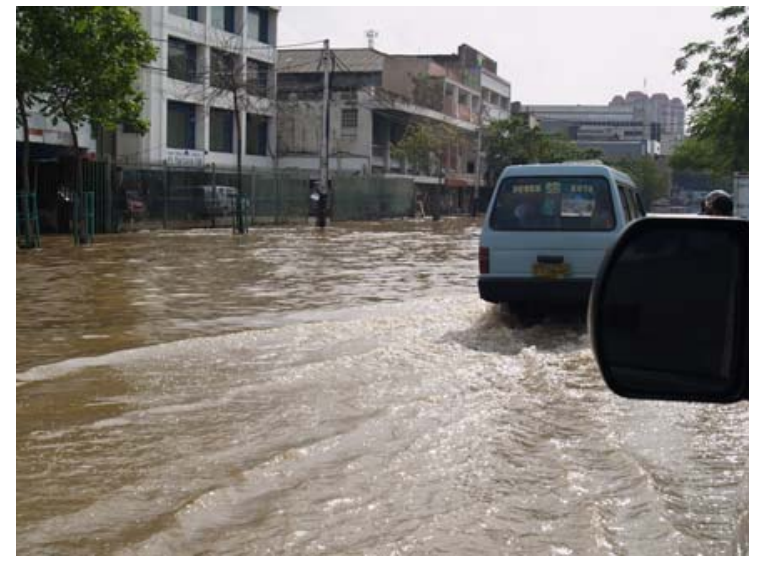

Fig. 1 Water is a key medium through which climate change impacts will be felt. As the global temperature increases, the hydrological cycle accelerates. The result will be changes in water availability-drought in some places and storms and floods in other. The melting of glacial ice is another concern; the glaciers in the Himalayas alone provide water for one-sixth of the world's population. Finally, rising sea levels present a challenge to coastal communities and low altitude islands around the world. (Jakarta 2007; Photo: Neil Chatterjee)

governance, financing and non-water changes in other agriculture, health and energy sectors Figs. 2, 3.

\section{The knowns...}

We know that there is climate change and we also know that the global population is growing and

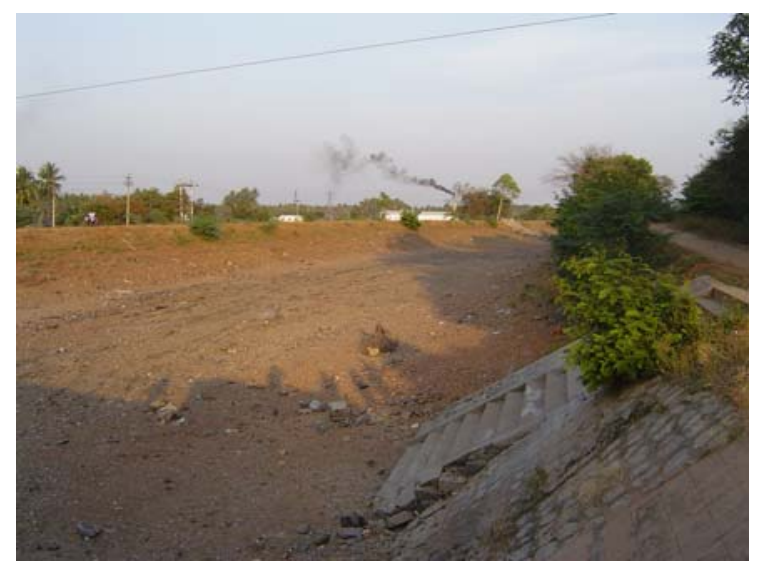

Fig. 2 Knowing where and how the impacts of climate change are most likely to affect populations and ecosystems through the water cycle will help in the identification of areas for early intervention. These particularly vulnerable areas and regions are commonly referred to as 'hot spots'. (India, Tamil Nadu 2004; Photo: Jan Lundqvist, SIWI)

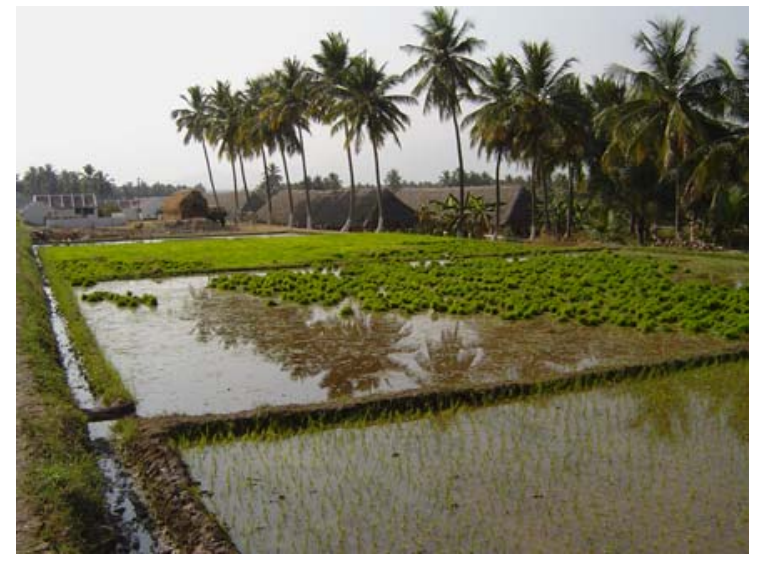

Fig. 3 The potential for increasing water stress is considerable and will have huge impacts on agricultural development across the globe in the future. Countries and regions will have to adapt to altered climatic conditions by e.g. switching to less water intense crops. (India, Tamil Nadu 2004; Photo: Jan Lundqvist, SIWI)

moving. The global system is not in a steady state. The changes in water availability and predictability of weather systems, along with population growth and movement put water at the heart of future development decision making. The potential for increasing water stress at local, national and even regional scales is considerable and will have huge impacts on agricultural development across the globe in the future. An agreement in COP-15 on mitigation and on adaptation is therefore crucial for water and for wider development-not least because adaptation decisions taken now will impact future mitigation strategies through shaping the way economies and societies develop. The measures put in place to manage, store, conserve and protect water resources now in the face of climate change will play a major role in shaping future human development.

Figure 4 At the Stockholm Water Week in August this year, a key text emerged in the form of a document now commonly called the Stockholm Statement. This was a call-a cry even-from the global water community present at the event to take water seriously within climate negotiations. However, not as a 'sector' in isolation (which water is not), rather as a fundamental issue at the heart of climate change issues that must be properly and adequately reflected within the COP-15 agreement and all follow-up negotiations. The message wasn't an isolated effort, but followed months of meetings 


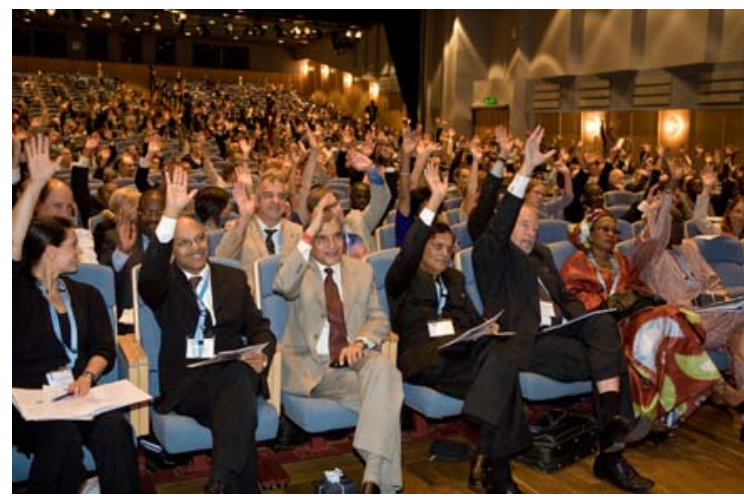

Fig. 4 In the closing plenary session of the recent 2009 Stockholm World Water Week participants unanimously voted to support the Stockholm Message on Water and Climate to the COP-15. This was a call from the gathered water community to the climate negotiators to properly acknowledge and address the water issue within the negotiations. The message stresses that water is the medium through which climate change will be expressed and that water and ecosystem management will be a key to successful climate change adaptation. (Stockholm Statement, World Water Week 2009; Photo: Mikael Ullén/ SIWI)

and activity, including the Dialogue on Climate Change Adaptation for Land and Water Management, the 5th World Water Forum in Istanbul and dialogues held at the Climate Change Negotiations in Bonn. The Stockholm Message incorporated much of this pre-existing discussion, synthesized it and presented it as a simple set of messages, stating that:

- Water is a key medium through which climate change impacts will be felt. Managing the resource effectively, including through well-conceived IWRM approaches and at a transboundary level, is central to successful adaptation planning and implementation, and to building the resilience of communities, countries and regions.

This is stating that we need to build on, reinforce and do better what we already do today. Improving on present, often poor, management and over-exploitation of the resource and improving the management of water services is an important step towards coping with climate change. Similarly, at scale, this also requires greater attention to shared water courses and aquifers.

- Adaptation is a prerequisite for sustainable development and poverty reduction. Adaptation measures thus need proper integration within broader development goals and objectives, including the Millennium Development Goals;

Responding adequately is not just about thinking within a sector or community, but is also about thinking how our response should be more collectively organized. This means ensuring that impacts really address poverty reduction and help to keep the global community on track to deliver against development goals and ensure that communities and countries build greater resilience to future shocks.

- Integration of water with land and forest management is key to effective adaptation. We strongly endorse the Nairobi Statement on Integrated Land and Water Resources Management for Climate Change Adaptation; we also emphasize that water-related adaptation can and should support global mitigation actions.

Mitigating global warming through land, forest and water management, in combination with measures to adapt to change, represents a powerful combination. Adaptation should increase the strength and range of mitigation actions. Key starting points should include water management and forest management inhabiting the same 'policy space' so that actions are integrated and one strengthens the other. Likewise, future land policy, including spatial planning (in rural, coastal and the urban environments) and land tenure issues are important factors to integrate with water planning and management.

- Ecosystem protection and sustainability is fundamental to adaptation and human development. We therefore urge increased efforts towards and investment in the protection and restoration of natural resources-including water-as an essential part of any adaptation process;

Natural resources are part of a natural infrastructure' that can play a key role in adapting to change. These systems are part of a natural feedback mechanism based on ecosystem responses. They require protection to ensure that detrimental negative or positive feedback loops are minimized, including those that lead to losses in biodiversity, or to reductions in natural offtake capacity, both of which can be critical to the livelihood security of communities. 
- Higher-quality information that is more effectively shared will strengthen responses. In particular there is a critical need for the water and climate communities to increase the sharing of information at all levels of policy and practicefrom global to local, and from local to global;

That we need to respond to change-but not necessarily knowing which responses are best and at which scale-poses major challenges for accessing and using knowledge. Central to this challenge is the need to ensure that knowledge is shared and not (inadvertently perhaps) hidden. Existing systems are in abundance to support sharing-above all the internet and current search engines-but their strengths could be enhanced through establishing more 'one-stop shops', which help to combine together key climate and water data. Progress in, for instance, downscaling climate models is one such area that needs careful support, particularly in making information more digestible to policy makers at a national level in developing countries; at the same time there is a need to ensure bottom-up sharing of local knowledge on current impacts.

- Vulnerability assessments and risk management are critical to sound adaptation practice. Knowing where and how the impacts of climate change are most likely to affect populations and ecosystems through the water cycle will help in the identification of areas for early intervention or 'hot spots'; these include arid regions, areas highly dependent on groundwater, small island developing states, low-lying deltas and fragile mountainous areas;

One such key knowledge area is our understanding of risk and vulnerability. Where are the impacts to be felt most acutely, and when? How can we then respond effectively in these 'hot-spot' areas? Simple combining of information sets that already exist is one step forward, particularly in highlighting what we already know about vulnerability and risk based on past data analysis. Above all, we need to know when situations of acute vulnerability have been reached and what consequences may follow in terms of loss of species and/or impacts on human and environmental systems.

- New and additional funds are essential. It is imperative that additional funding is allocated in support of developing adaptive strategies for vulnerable groups and ecosystems; there is a need for an initial mobilization of finance to assist vulnerable, low income countries already affected by climate change, followed by the establishment of a well-resourced mechanism for funding adaptation as part of ongoing climate negotiations.

Information development and management is one area where future funding is important, not least because many developing countries require stronger capacity in this area. But it is also important that in identifying the most vulnerable communities and countries, the next step becomes of the provision of support and assistance to their responses to climate change, both immediately and in the longer term. Stronger institutions and institutional cooperation at all levels between the climate, water and wider development communities is a basic foundation of better adaptation measures and requires stronger financial foundations. Such funding mechanisms should be governed democratically and efficiently and provide the necessary flexibility to cater for a variety of needs. At a national level, countries must be able to receive and allocate funds from multiple sources with minimal transaction costs, and ensure that local government and organizations have access to the resources they require for sustainable responses at a local level.

\section{Follow up}

This is not just a wish-list or to-do list for policy makers and negotiators. These are simple, common-sense responses to existing knowledge levels, as well as a call to find more out about existing knowledge areas and to identify things possible 'unknown unknowns' before they are encountered unexpectedly.

This is also a call to develop a global community in which water makes up one part of major collective action on addressing the multiple-and multiplying - climate challenges ahead. It is also a call for a vision of what to do beyond COP-15, in order to strengthen existing global mechanisms through greater dialogue between the Water and Climate communities. 